\title{
Enzyme-Catalyzed in situ Synthesis of Temporally and Spatially Distinct CdSe Quantum Dots in Biological Backgrounds.
}

Ryan A. Riskowski ${ }^{\dagger}$, Richard S. Nemeth ${ }^{\dagger}$, Kanda Borgognoni, Christopher J. Ackerson*

Department of Chemistry, Colorado State University, Fort Collins, Colorado, 80524 under

${ }^{\dagger}$ Both of these authors contributed equally to this work.

KEYWORDS: Biogenic Nanoparticles, Quantum Dots, Enzyme Synthesis

\section{CONTENTS}

1. XRD of CdSe Nanoparticles 1

2. Absorbance Spectra of CdSe Reactions in LB........................................................ 2

3. HR-TEM Histograms and Fits of CdSe Diameters $w \& w / o$ GRLMR . 4

\section{XRD of CdSe Nanoparticles}

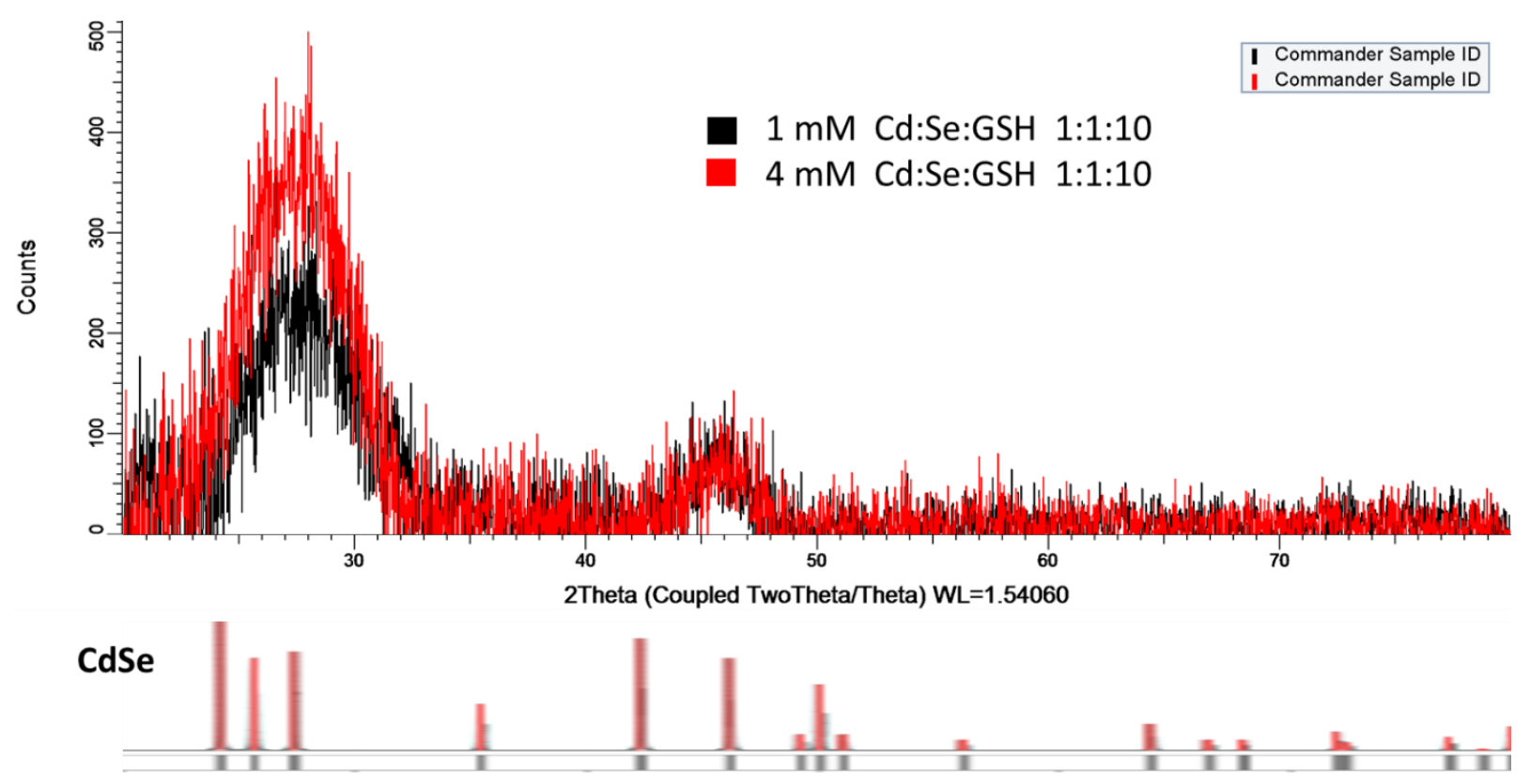

Figure S1. XRD results showing the formation of CdSe crystal structure. Synthesis was conducted in LB with 10x excess GSH relative to selenite. Red and black lines show results for synthesis using $4 \mathrm{mM}$ or $1 \mathrm{mM}$ selenite respectively. Cadmium was added at a 1:1 ratio with Selenium. 


\section{Absorbance Spectra of CdSe Reactions}

Absorbances were collected from $290-750 \mathrm{~nm} 1$ hour after reactions were mixed, and show the observable formation of CdSe quantum dot exciton absorbance.

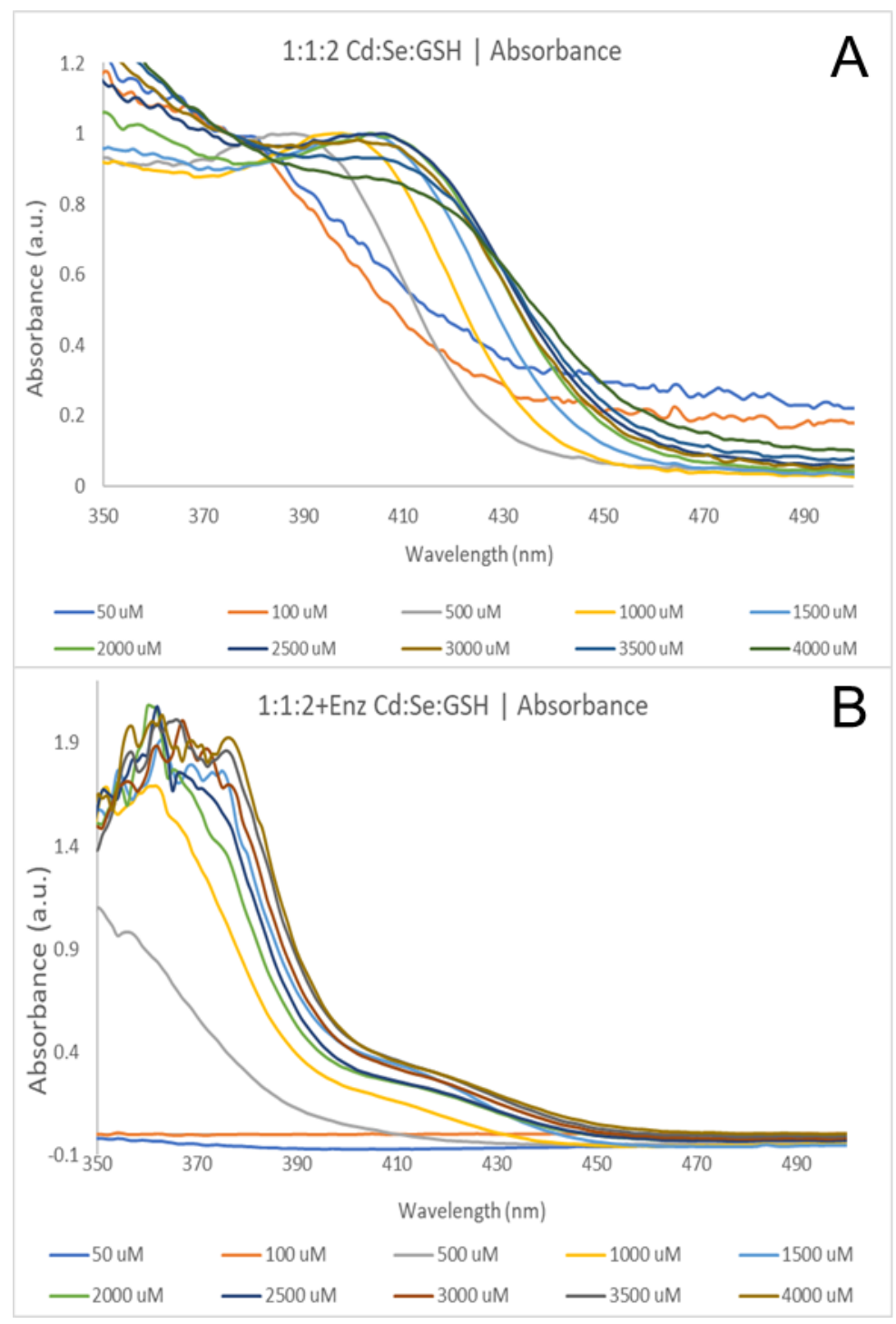

Figure S2. Absorbance of CdSe in LB, using 2 molar equivalents of GSH without enzyme (A) or with enzyme (B). The persistent absorbance of NADPH (added only in reactions containing enzyme) is due to a rapid depletion of substrate (selenodiglutathione). The substrate begins at very low levels in these reactions because the two molar equivalents of GSH must be shared by selenite and cadmium to form our precursor materials (selenodiglutathione and cadmium-glutathione chelate). The overlapping absorbance of NADPH and CdSe particles in Figure S2B makes it difficult to ascertain the true position of the exciton peak ( $410 \mathrm{~nm})$. 


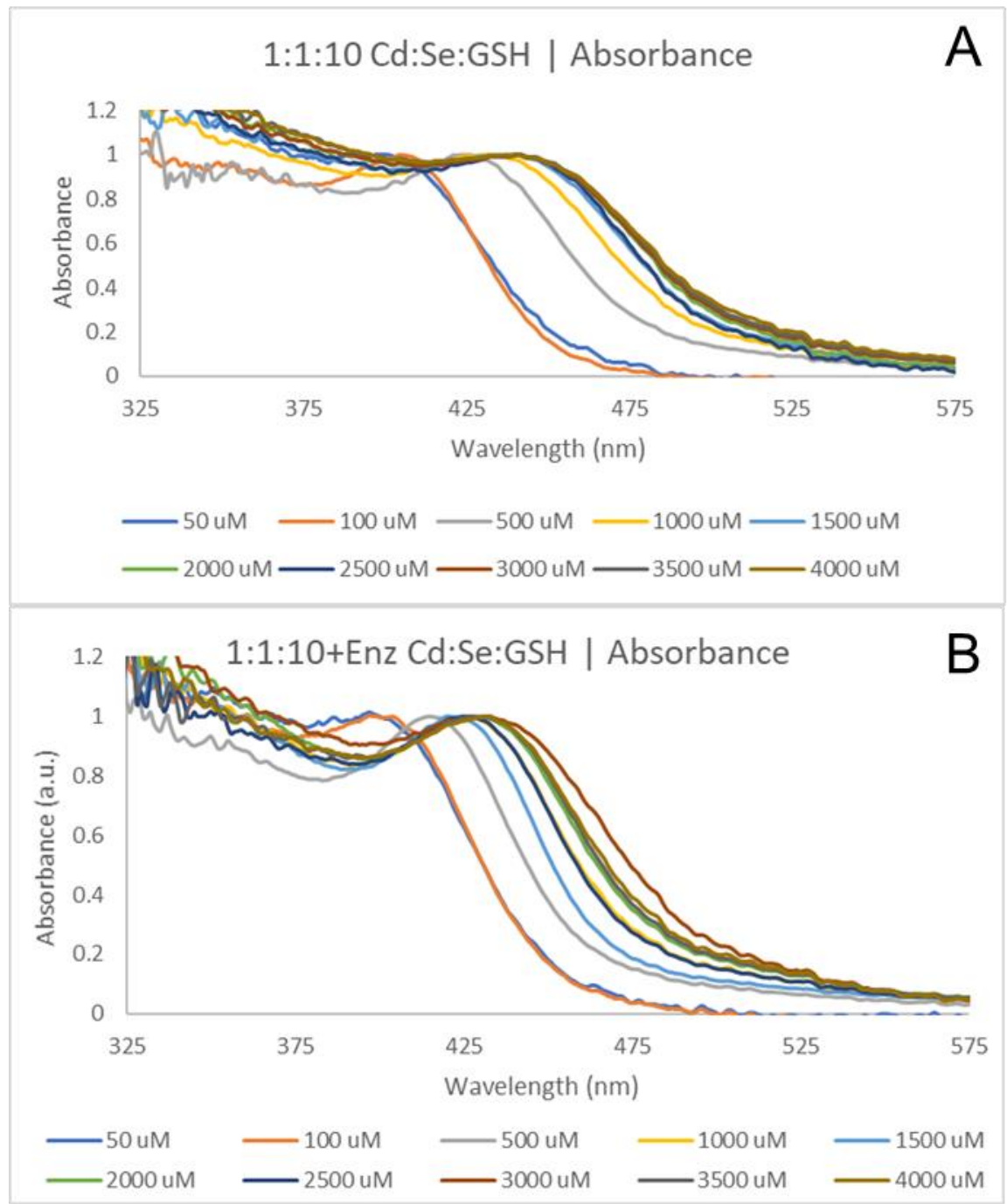

Figure S3. Absorbance of CdSe in LB, using 10 molar equivalents of GSH without enzyme (A) or with enzyme (B). Comparison of the exciton peaks demonstrate a blue-shifted peak absorbance for reactions with enzyme (B) as compared to the absence (A). 


\section{TEM Histograms and Fits of CdSe Diameter w/ \& w/o GRLMR}

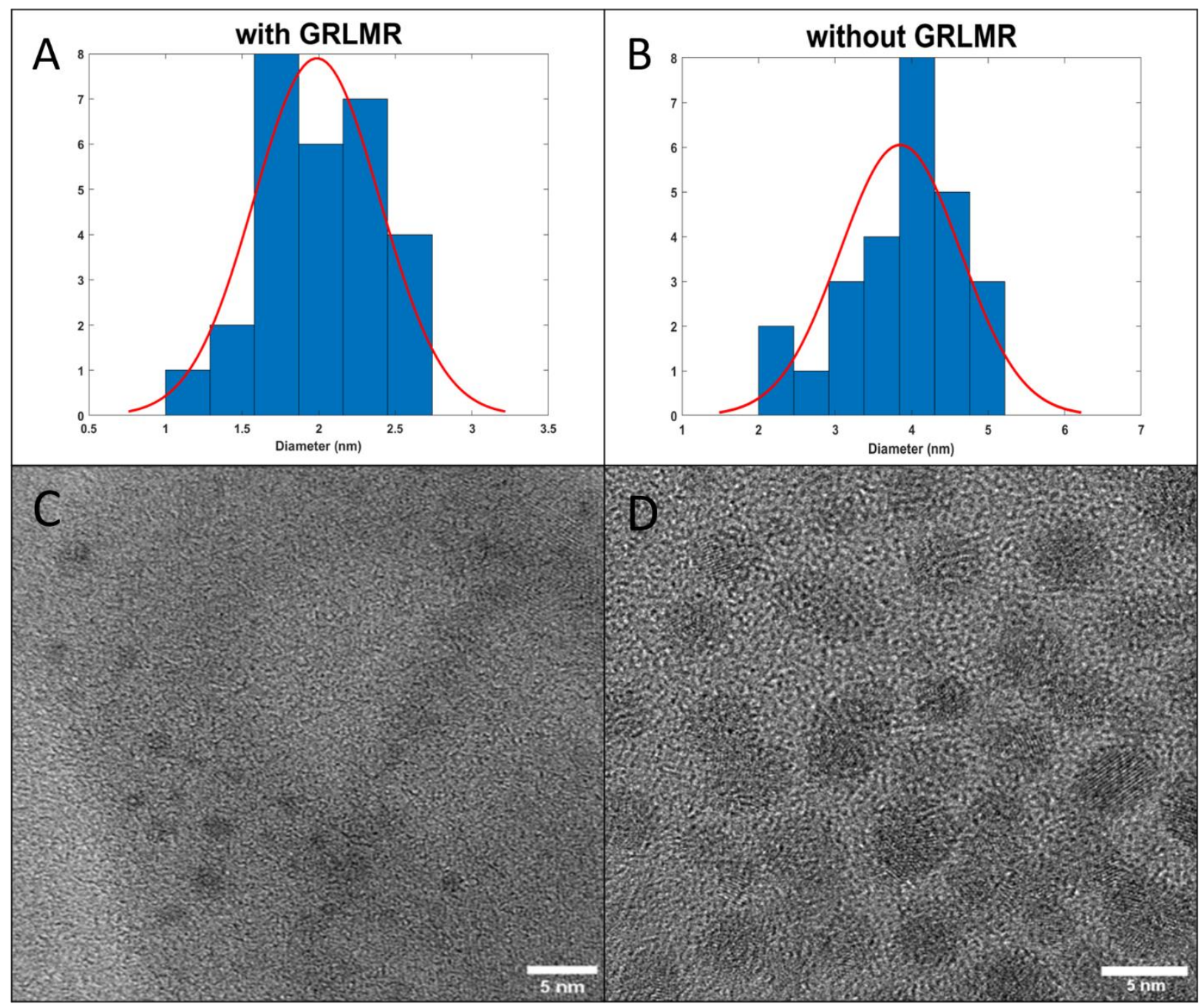

Figure S4. Histograms showing the diameter of (A) GRLMR \& (B) GSH formed nanoparticles as measured by HR-TEM (C) GRLMR formed \& (D) GSH formed. The normal fits reveal that particles formed by GRLMR enzyme are smaller diameter $(\mu=2.0 \pm 0.4 \mathrm{~nm}$ ) with reduced polydispersity (FWHM $=0.97 \mathrm{~nm}$ ) compared to particles formed with GSH alone $(\mu=3.9 \pm 0.8 \mathrm{~nm})$ and (FWHM $=1.86 \mathrm{~nm}$ ). Samples were prepared by mixing $200 \mu \mathrm{M}$ Selenite with $100 \mu \mathrm{M}$ cadmium acetate and 10x molar equivalent of GSH in 1x PBS. Two batches were prepared, one with GRLMR and one without GRLMR. Both reactions contained $400 \mu \mathrm{M}$ of NADPH to ensure that only the presence or absence of the enzyme determined the outcome of particle formation. Immediately after the reaction, aliquots were dropped onto 200 mesh carbon-coated copper grids pretreated by glow discharge under O2. Samples were then imaged with a $200 \mathrm{kV}$ JEM 2100 HR-TEM using a 30cm camera length. Particles were sized in ImageJ using a minimum of 30 particles per dataset. Histograms and fits were generated in MATLAB. 